Baramidze T,

Mardaleishvili K.,

Oncology Scientific-Research Center of Georgia

E-mail: tamarbaramidze5@gmail.com

\title{
RESULTS OF COMBINATION THERAPY FOR SQUAMOUS CELL CARCINOMA OF THE MAXILLA
}

\begin{abstract}
Surgical treatment of these patients is very important - After post-operative treatment, complicated prosthesis using dental implants. Which is the goal of our article.
\end{abstract}

Keywords: combination therapy, dental implants, prosthesis using, oncological diseases, maxillary cancer, primary and secondary maxillary.

Upper jaw cancer, Squamous Cell Carcinoma, Postoperative rehabilitation, Complicated prosthesis, Dental implants.

Malignant tumors of the maxilla make up $2-4 \%$ of all oncologic diseases and are equally common in men and in women. Although this pathology mainly occurs in patients aged 40 years and over, it also may be encountered in children and young people. Most frequently the tumorous process develops from the maxillary sinus epithelium. In the second and third place are ethmoid labyrinth tumors followed by those originating from the tissues of the nasal cavity.

Significant etiological factors of maxillary malignant tumors can be chronic inflammation of the maxillary sinuses, trauma of these structures etc. Depending on the onset of the disease, primary and secondary maxillary and mandibular malignant tumors have been classified.

Primary tumors originate in the bony tissue, while secondary malignancies involve tumors that develop from the ethmoid sinus of the maxilla, soft and hard palate and the mucous membrane of the alveolar process.

Maxillary cancer is characterized by a diverse symptomcomplex - dull, persistent pain, difficult nasal breathing or purulent bloody nasal discharge, lacrimation, exophthalmos, and swelling of facial soft tissues.

In maxillary cancers, not all of these symptoms manifest themselves simultaneously, which can be explained by their complicated topographic and anatomical features.

42 patients diagnosed maxillary malignant tumor underwent treatment in Konstantine Mardaleishvili Medical Center (Oncology Scientific-Research Center of Georgia) within the period of 2014-2019. Of them 29(69.8\%) were men, 13(30.2\%) - women. 7 (18.8\%) patients aged 40, 33(78.9\%) patients over the age of 40, 2 (4.7\%) cases under 20. Malignant tumor of the right half of the maxilla was revealed in 19(4.5\%), left half of the maxilla- $15(3,9 \%)$ patients. Malignant tumor of the maxillary central incisors area (bilaterally) was found in $8(1.9 \%)$ patients. The duration of disease for 6 months was observed in $16(36.2 \%)$ patients, for one and a half year and over- in $26(62.8 \%)$ patients. 4 patients associated their illness with cystic neoplasm, 6 (18.8\%) patients had a history of chronic rhinosinusitis with recurrent acute exacerbation, $3(8.1 \%)$ patients associated their illness with a tooth extraction, 7(19.4\%) -with the use of completely disposable dental prosthesis, the rest of the patients could not indicate the cause.

Diagnosis making in maxillary cancer is rather difficult. Diagnostic errors due to the numerous symtomocomplexes and complex topographic and anatomical features of the disease are not rare. Therefore, if a malignant tumor is suspected, it is necessary to conduct a consistent clinical and morphological study, starting with a visual examination of the patient's face with a proper assessment of the condition of nasolabial wrinkle and the nasal breathing on the affected side.

$33(89 \%)$ of the patients admitted to the Center received fibroscopic examination, $9(11 \%)$ underwent fibroscopy with targeted biopsy. In 21(48.1\%) cases squamous cell carcinoma was identified.

The material for cytological examination was collected not only endoscopically, but also with the help of puncture, trepanation, and also through the analysis of flush water during the irrigation of the cavity (3\%).

A morphological study of the surgical material of all patients was carried out. Squamous cell carcinoma was detected in $21(48.1 \%)$ patients, osteogenic sarcoma $-5(12.1 \%)$, cylindroma $-14(33.1 \%)$, tumors of various origins $-2(4.8 \%)$.

Radioisotopic examination was carried out on $4(9.8 \%)$ patients.

Computed Tomography provides an accurate imaging of cartilaginous, muscular, fatty, and other tissues, enabling to determine the tumor growth tendency and the degree of bony structures destruction. This method also makes it possible to clarify the boundaries of the tumorous tissue, as well as interdependence of the tumor with soft and bony structures.

$42(100 \%)$ patients received computed tomography and magnetic resonance examination. Based on the examination results tumor spread to the orbital area was observed 
in $7(14.6 \%)$, pterygopalatine fossa $-4(9.2 \%)$, infratemporal and temporal fossa $-2(5 \%)$, hard and soft palate $-24(5.9 \%)$, lacrimal duct $-5(14.8 \%)$ cases. In all the ases tumor spread to the alveolar process was observed.This, based on the TNM Classification we revealed and established the following: Stage 1-9 (21.4\%), Stage 2-15 (37.7\%), Stage 3-12 (28\%), Stage 4-6 (15\%) For the detection of regional metastases the patients received ultrasound examination of cervical lymph nodes; hematogenic metastases were identified using computed tomography of the lungs.Based on the above, regional metastases were revealed in $10(23.7 \%)$ cases, hematogenic metastases $-2(5 \%)$, in 30(72\%) cases no regional metastases were observed.According to the clinical and Computed Tomography findings as well as the results of cytological study, an individual method of treatment was scheduled for each patient.

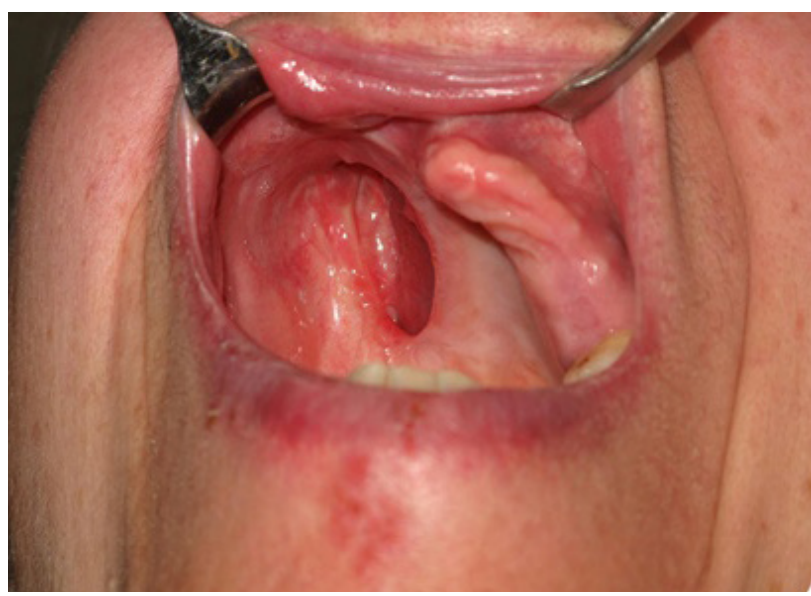

The slide shows the postoperative defect after 6 months and the defect and teeth alignment restored by complex prosthetic surgery with the purpose to significantly reduce postoperative deformation of the face, restore the integrity of the teeth alignment, and to detach the oral cavity from the upper respiratory organs, so that the patients could eat and resume their chewing, swallowing and respiratory functions.

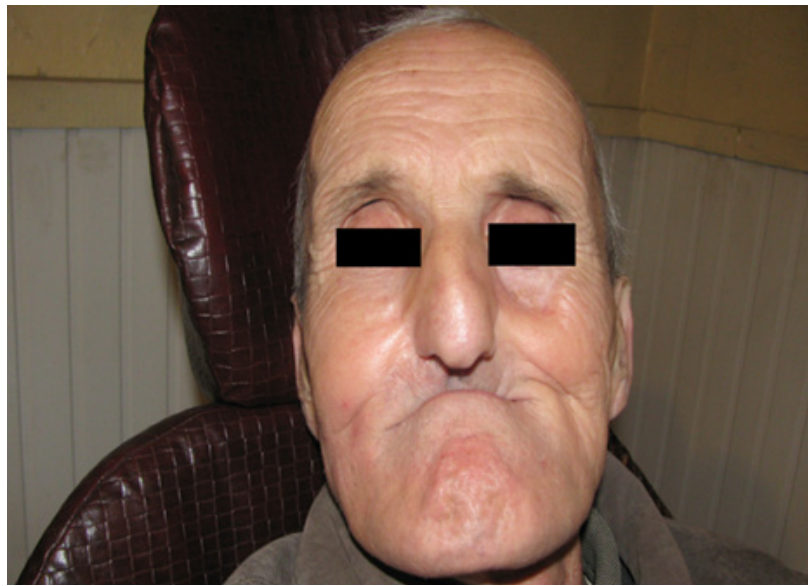

Consequently, combined treatment method was planned for 38 (90\%) patients, radiation therapy as a monotherapy method for 4 (9,8\%) patients; recurrence after radiation therapy was revealed in $4(9,8 \%)$, postoperative recurrence - in $6(11.8 \%)$ patients

Surgical treatment: Resection of the right half of the maxilla-28(69,7\%) cases, Resection of the left half of the maxilla $-14(30.3 \%)$ cases, Resection of the maxilla, orbitotomy $7(14.6 \%)$ cases,

Resection of the maxilla, exenteration $-3(6.7 \%)$ cases, Resection of both halves of the maxilla $-2(5 \%)$ cases, Resection of the maxilla, lymphadenectomy $-17(45,1 \%)$.

Before surgical treatment, all patients received orthopedic treatment using a separating plate for partitioning the oral cavity from the operated defect. 6 months after the surgical treatment, patients underwent complex prosthetic surgery.

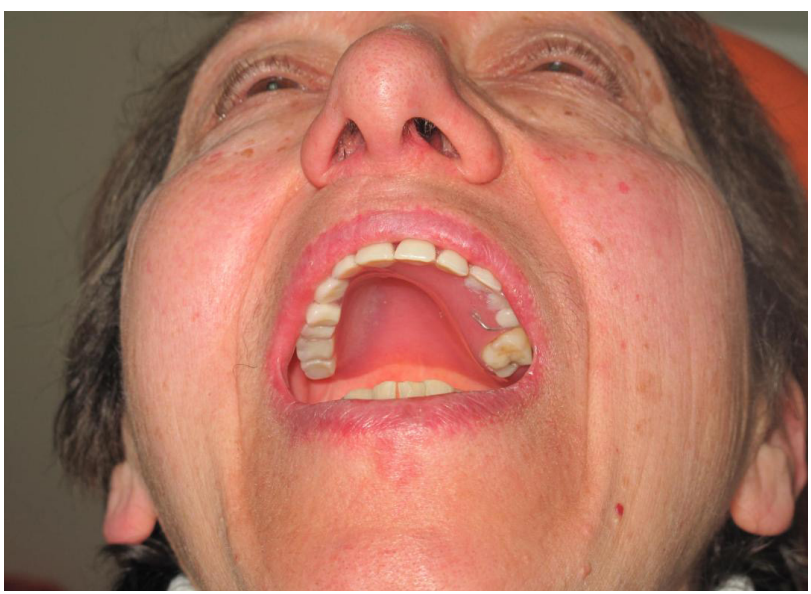

For rehabilitation purposes, after the resection of the right and left halves of the maxilla, dental implants were placed in 3 patients, which enabled to perform a complex prosthetic surgery. The slide shows the photo of the patient after the resection of both (right and left) halves of the maxilla.

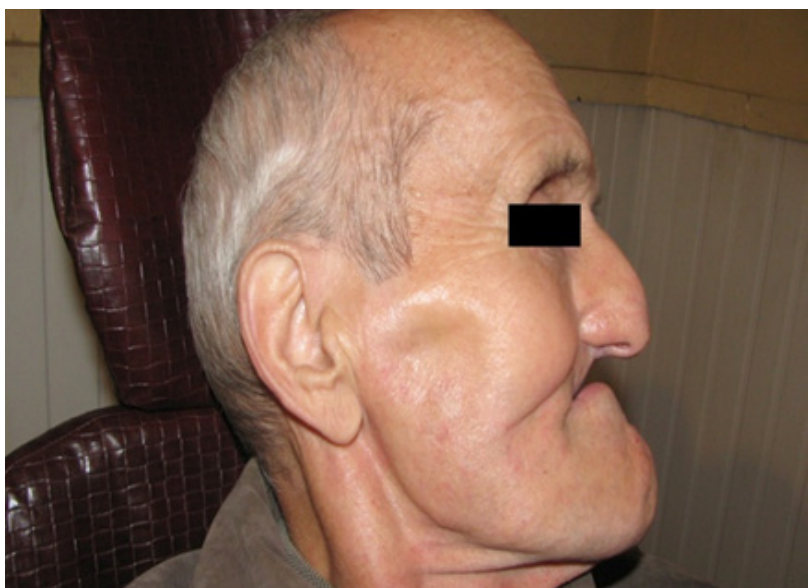




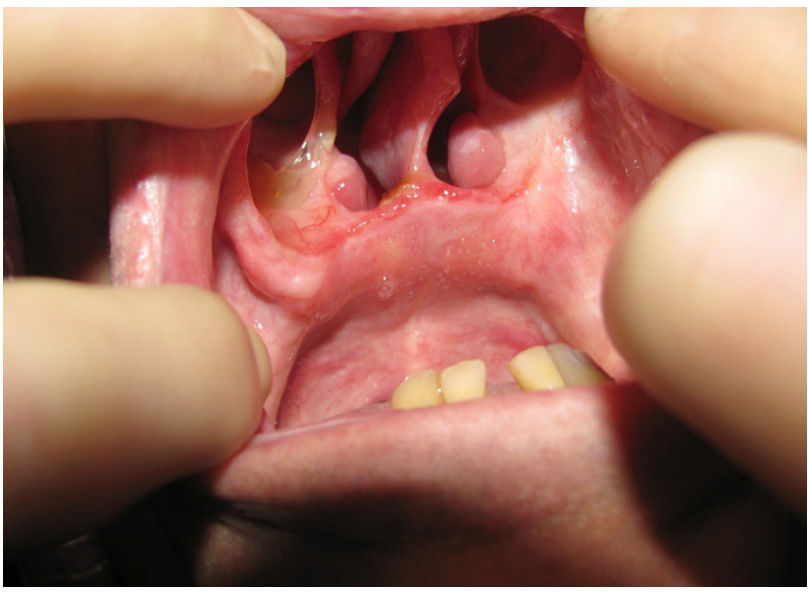

The slide shows the patient's oral cavity after the resection of both halves of the maxilla.

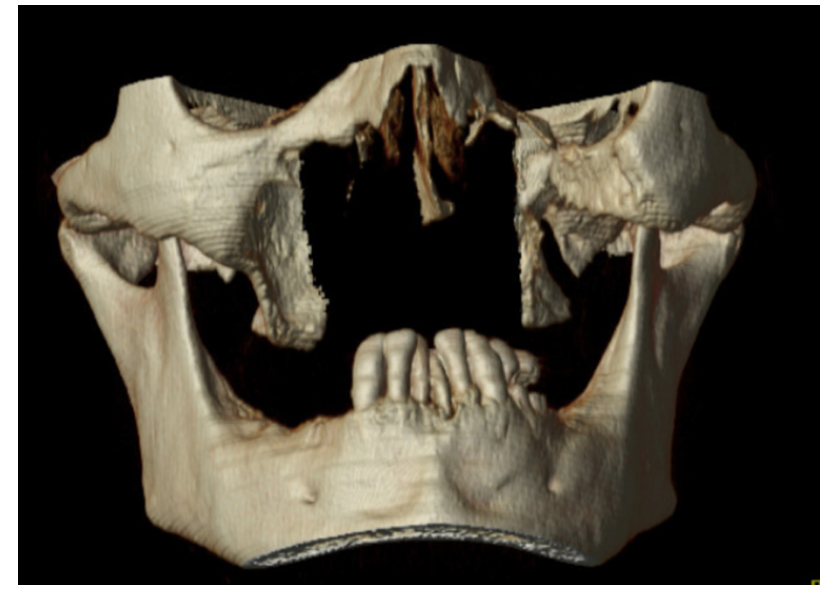

The orthopantomograph shows the two dental implants fixed into the rest of the jaw bone.

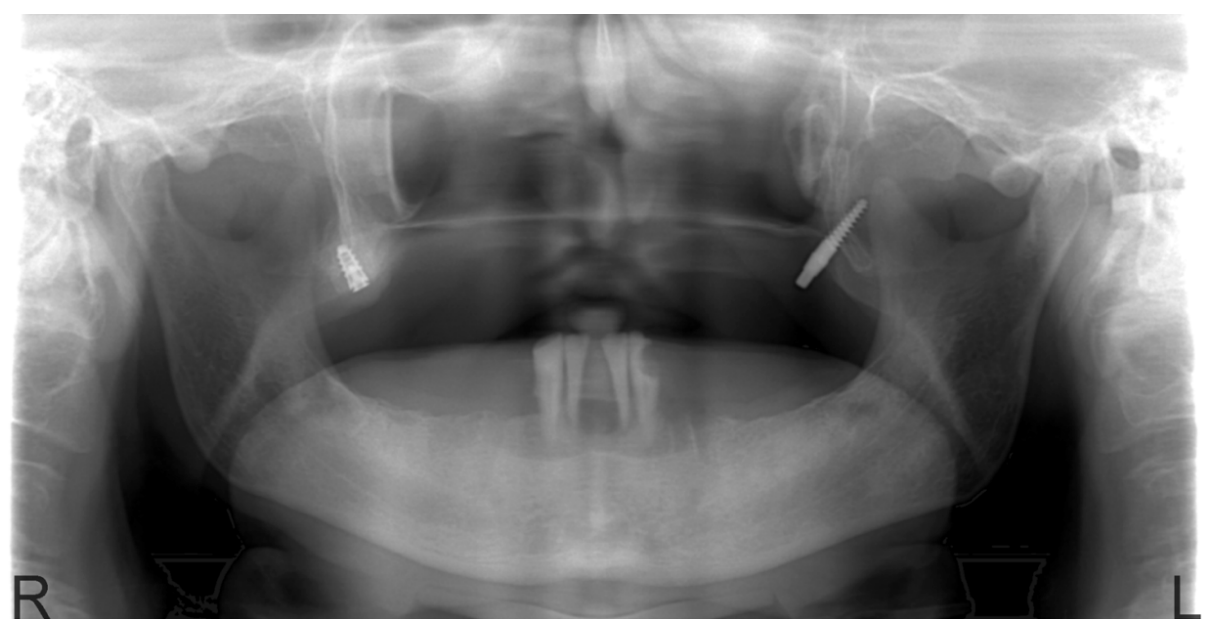

The slide shows metal ceramic crowns attached to the dental implants for the fixation of completely disposable prosthesis to achieve the orthognatic occlusion.
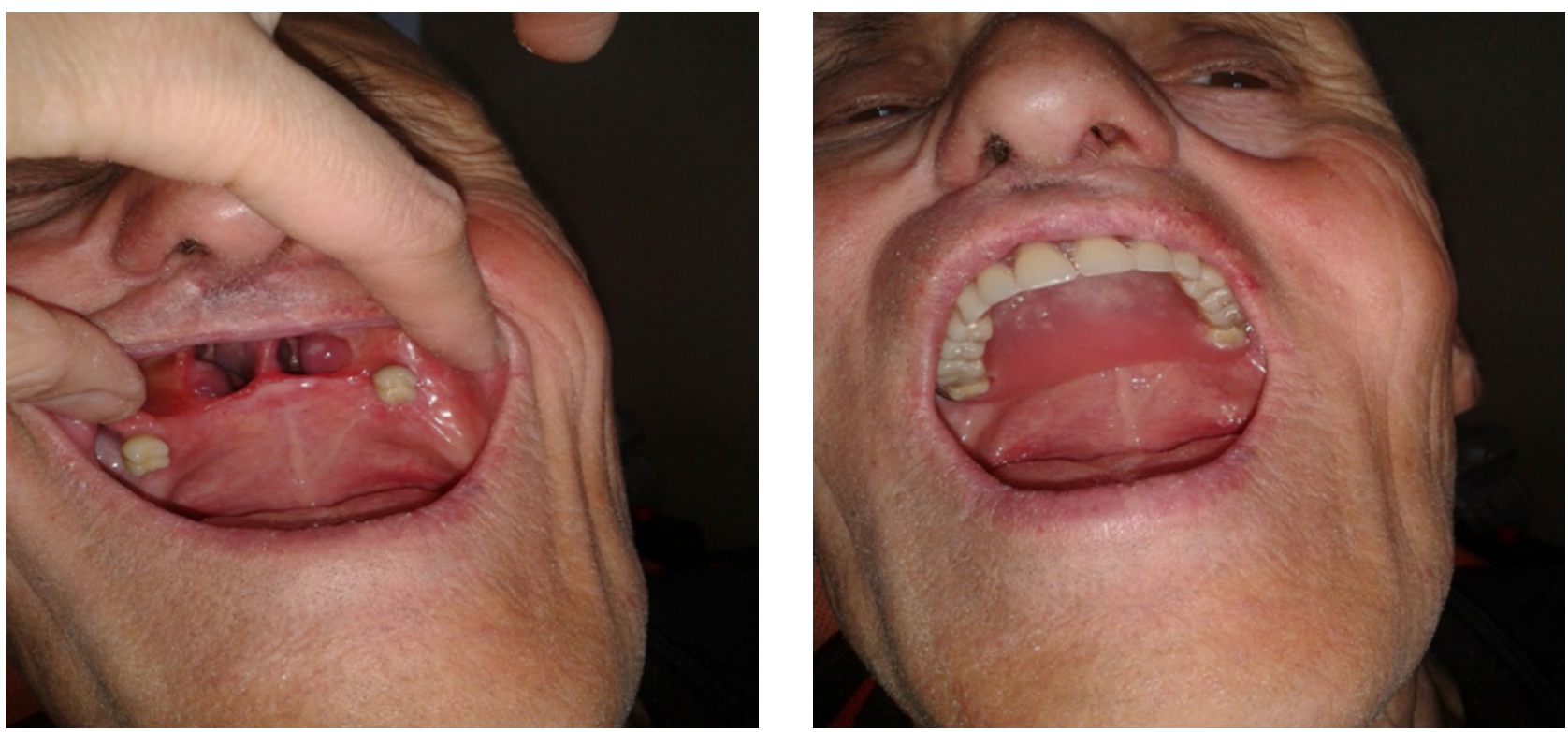


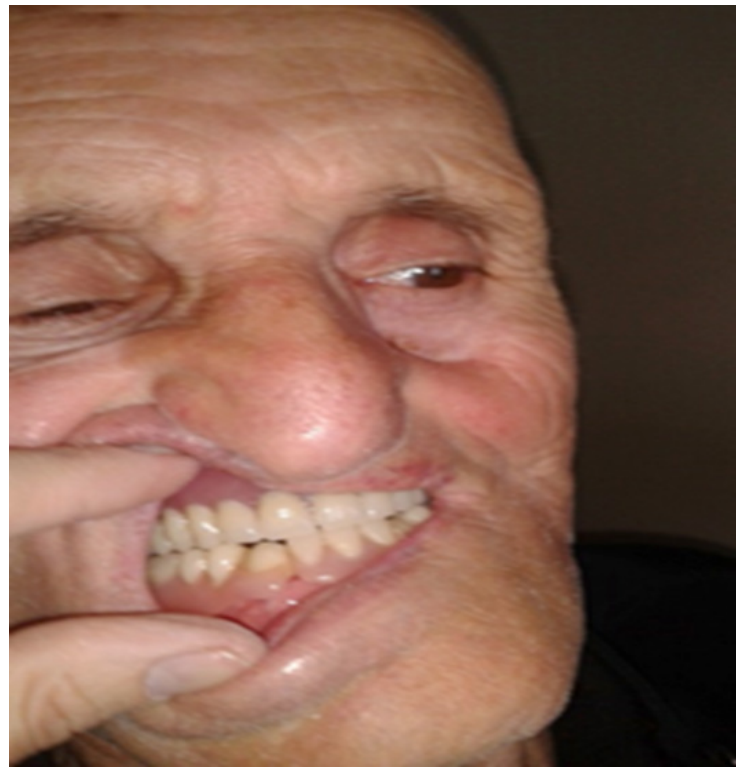

The following slide shows the patient before and after the complex orthopedic plastic surgery.

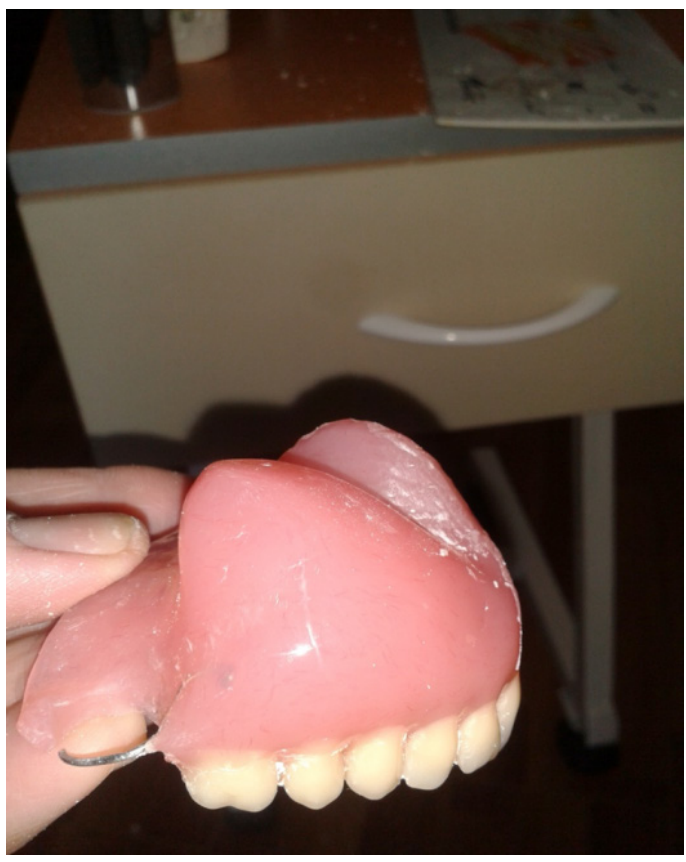

Out of 42 patients with squamous cell carcinoma, $31(73,9 \%)$ patients have survived, living for 3 years now. 3 $(7,21 \%)$ patients died in a year and a half after being diagnosed, due to the rapid progression of the disease. $2(5 \%)$ patients had a relapse of the disease and received relevant treatment; they have been alive for a year after the treatment. The fate of 3 (7.21) patients is unknown. 2 (5\%) patients are currently undergoing chemotherapy due to the progression of the disease.

\section{Summary:}

Maxillary tumors make up 2-4\% of all oncological diseases and $24 \%$ of head and neck tumors. This pathol-
In one case, there was self-rejection of the dental implant due to the progression of the tumor process.

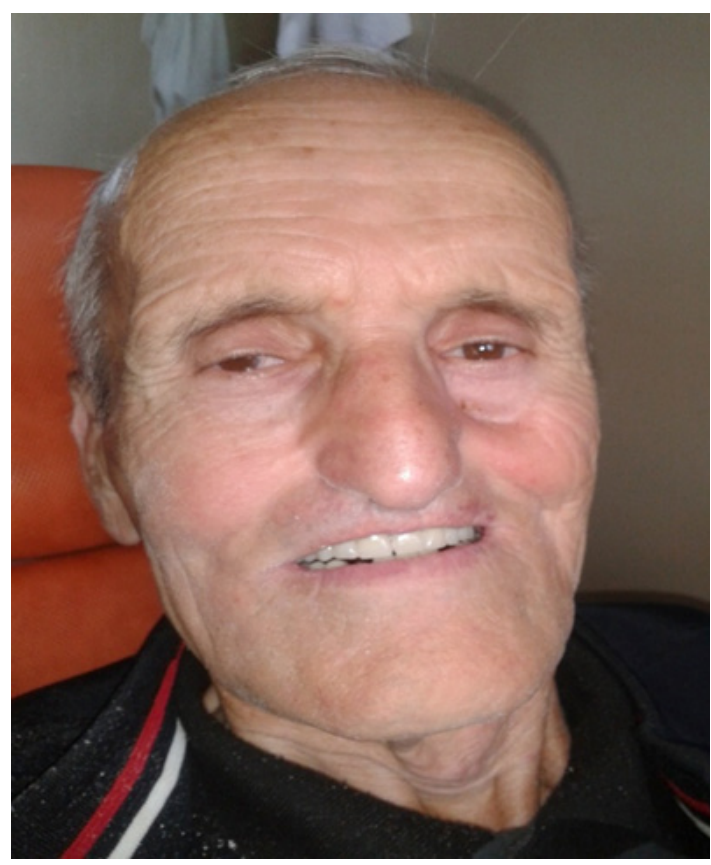

ogy is equally common both in men and women patients, aged above 40 years. Maxillary cancer was first discovered in the human skull of stone age. Famous representatives of the medical field, such as Freud and Pirogov were suffering from maxillary cancer. Upper jaw cancers are characterized by numerous symptoms. This symptomocomplex can be neurological, ophthalmic, otorhinolaryngological and dental. To study the maxillary cancer it is important to consider (reriew) the relationship between the upper jaw and neighboring bones. For this purpose, we will briefly refer to the skull. 


\section{References:}

1. Mardaleishvili K., Baramidze T. Malignant Tumors of the Face and Jaws.- Tbilisi, 2008.

2. Paches A.I. Tumors of the Head and Neck, 2002.

3. Eugene N. Myers Saunders. Cancer of the head and neck. 2003.

4. Otolaringology and Facial plastic surgery.URL: http://www.emedicine.com

5. American Dental Association. URL: http://www.ada.org 